\title{
Lack of color integration in visual short-term memory binding
}

\author{
Mario A Parra • Roberto Cubelli • Sergio Della Sala
}

Published online: 10 May 2011

(C) Psychonomic Society, Inc. 2011

\begin{abstract}
Bicolored objects are retained in visual short-term memory (VSTM) less efficiently than unicolored objects. This is unlike shape-color combinations, whose retention in VSTM does not differ from that observed for shapes only. It is debated whether this is due to a lack of color integration and whether this may reflect the function of separate memory mechanisms. Participants judged whether the colors of bicolored objects (each with an external and an internalcolor) were the same or different across two consecutive screens. Colors had to be remembered either individually or in combination. In Experiment 1, external colors in the combined colors condition were remembered better than the internal colors, and performance for both was worse than that in the individual colors condition. The lack of color integration observed in Experiment 1 was further supported by a reduced capacity of VSTM to retain color combinations, relative to individual colors (Experiment 2). An additional
\end{abstract}

Electronic supplementary material The online version of this article (doi:10.3758/s13421-011-0107-y) contains supplementary material, which is available to authorized users.

M. A. Parra $\cdot$ S. Della Sala

Human Cognitive Neuroscience and Centre for Cognitive

Ageing and Cognitive Epidemiology, Psychology Department,

University of Edinburgh,

Edinburgh, UK

M. A. Parra $\cdot$ R. Cubelli

Department of Cognitive Sciences and Education,

University of Trento,

Trento, Italy

R. Cubelli

Centre for Mind/Brain Sciences, University of Trento,

Trento, Italy

M. A. Parra $(\bowtie)$

Human Cognitive Neuroscience, Psychology,

University of Edinburgh,

7 George Square,

Edinburgh EH8 9JZ, UK

e-mail: mprodri1@staffmail.ed.ac.uk account was found in Experiment 3, which showed spared color-color binding in the presence of impaired shape-color binding in a brain-damaged patient, thus suggesting that these two memory mechanisms are different.

Keywords Memory binding · Short-term memory Working memory Associative memory

\section{Introduction}

Visual short-term memory (VSTM) represents multifeature objects either as integrated units (Luck \& Vogel, 1997; Vogel, Woodman, \& Luck, 2001) or as sets of individual features (Wheeler \& Treisman, 2002). Some authors have found that unifeature and multifeature objects can be retained in VSTM with similar efficiency (e.g., Brockmole, Parra, Della Sala, \& Logie, 2008; Gajewski \& Brockmole, 2006; Luck \& Vogel, 1997; Vogel et al., 2001), suggesting that multifeature objects are represented in VSTM as integrated units and that their binding is costfree (i.e., object-based format). Other authors have shown that VSTM performance for multifeature objects is significantly poorer than VSTM performance for unifeature objects (e.g., Olson \& Jiang, 2002; Parra, Abrahams, Logie, \& Della Sala, 2009; Parra, Della Sala, Logie, \& Abrahams, 2009; Wheeler \& Treisman, 2002; Xu, 2002), suggesting that VSTM represents individual features and that the binding between these features requires additional memory capacity (i.e., feature-based format).

These different results seem to depend on the kind of features to be bound, which could belong either to the same dimension or to different dimensions. Typically, studies showing no differences have compared VSTM performance for object-color bindings and objects only (Brockmole et al., 2008; Gajewski \& Brockmole, 2006; Vogel et al., 2001). In contrast, studies reporting differences have compared VSTM for color-color bindings and single colors (Olson \& Jiang, 2002; Parra, Abrahams et al., 2009; Parra, 
Della Sala et al., 2009; Wheeler \& Treisman, 2002; Xu, 2002).

One way to reconcile this discrepancy is to posit that resources drawn from a single dimension would be depleted quickly (i.e., more interference), whereas resources drawn from different dimensions would be shared and memory operations would be less constrained by capacity limitations (Olson \& Jiang, 2002; Wheeler \& Treisman, 2002). However, the observation that retaining bicolored objects in VSTM requires more memory capacity than does retaining unicolored objects does not rule out the possibility that colors are integrated within single units. For example, Parra, Abrahams et al. (2009); Parra, Della Sala et al. (2009) used a change detection task to measure recognition of bicolored objects across study and test displays. In one condition, one color of two bicolored objects was replaced by a new color in the test display. Hence, memory for the individual colors presented at study was enough to perform the task. In a second condition, one color of two bicolored objects was swapped between objects at test; hence, the way the colors were combined at study (i.e., binding) was the crucial piece of information. Participants performed the former condition significantly better than the latter (Parra, Abrahams, et al., 2009; Parra, Della Sala, et al., 2009). The only difference between these two conditions is the additional requirement in the second condition to remember how colors combine. These results do not rule out the possibility that color combinations can be represented in VSTM in an object-based format. They suggest only that, in doing so, more resources might be required.

Gajewski and Brockmole (2006) distinguished between the object-unit hypothesis and the independent-stores hypothesis. The object-unit hypothesis assumes that the proportion of trials on which participants report both features correctly is a function of the probability of remembering the item (whole unit) and the probability of guessing both features correctly when the item is not remembered. From this perspective, the item would be remembered only if information about both constituent features is accessible in memory. On the contrary, the independent-stores hypothesis assumes that features can be remembered and guessed independently. Therefore, there should be no need for representing the object as a whole in order to have access to its constituent parts. They found that when people have to retain objects defined by shape-color bindings in VSTM, they can remember the objects only if they correctly remember both constituent features (i.e., an all-or-none principle). Hence, remembering only one feature does not grant access to the object representation. In the unified object representation, once features are bound, they lose their individual properties and adopt the properties that define the new object. Gajewski and Brockmole investigated VSTM only for shape-color bind- ings. Therefore, it is not known whether color binding leads to the formation of new object representations and whether this occurs following the all-or-none principle.

Previous studies on color-color binding in VSTM (Olson \& Jiang, 2002; Parra, Abrahams et al., 2009; Parra, Della Sala et al., 2009; Wheeler \& Treisman, 2002) have not permitted the identification of the type of format used to represent color combinations in VSTM. This is because the tasks used in these studies did not allow ascertaining whether memory for color parts of bicolored objects yielded different behavioral results. Taken together, the approach proposed by Gajewski and Brockmole (2006) and the methodology used by Parra, Abrahams, et al. might enable the investigation of whether or not colors are bound into integrated units in VSTM. Parra, Abrahams, et al. used a change detection task that presented bicolored objects in two conditions that differed only in their binding requirement. Because any changes occurred in the same part of two of the objects (i.e., internal or external colors), this design might allow the assessment of memory for objects' parts, should this principle apply to bicolored objects (instead of being remembered as integrated units).

The independent-stores hypothesis would predict a greater cost for representing combined colors in VSTM than for representing the same number of individual colors (see Fig. 1; $\mathrm{AB}>\mathrm{A} \& \mathrm{~B}$ ).Because colors are represented in VSTM not as integrated units but as individual entities, retaining how colors combine would require extra memory capacity. If this were the case,each color of bicolored objects could be differentially remembered, whether they have to be remembered together or individually. The rationale is that if color parts of bicolored objects are not bound into unified representation, they will be retained separately in VSTM, and this may lead to different patterns of errors when either part changes. Alternatively, if colors are bound in a way that leads to the formation of new objects, memory accuracy for each individual color will not differ, since changing either color will result in a new object (as predicted by the objectunit hypothesis). Experiment 1 is set up to determine which of these two hypotheses better accounts for VSTM performance during feature binding within the color dimension (see Fig. 1 for experimental hypotheses).

\section{Experiment 1}

A novel change detection task was used to investigate whether VSTM performance for bicolored objects would differ when changes occurred in either of the two colors and when colors had to be remembered together or as individual features. This design enabled the assessment of the type of representation used to retain color combinations in VSTM,since memory for each color part could be separately measured. This permittedus 
Fig. 1 Hypotheses assessed in Experiments 1-3. Experiment 1 investigated whether color combinations could be retained in visual short-term memory (VSTM) as integrated objects or as individual features that are associated within objects. Experiment 2 investigated whether the additional cost of retaining color-color binding in VSTM $(\mathrm{AB}>\mathrm{A} \& \mathrm{~B})$ holds when the number of to-be-remembered objects increased. Experiment 3 assessed the hypothesis that if colors are not bound into unified representations, this memory function should dissociate from that supporting shape-color binding,since the last one does lead to integrated objects in VSTM. This hypothesis was tested in a patient who had previously shown severe impairment in the latter function

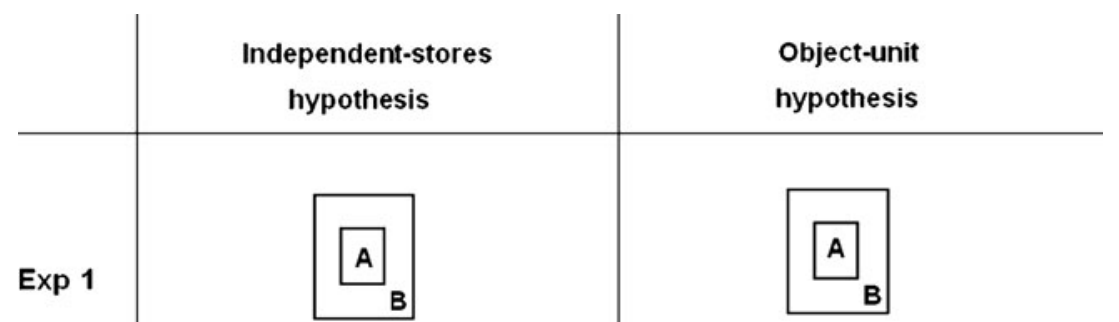

Prediction 2: $\quad A B=A \& B$

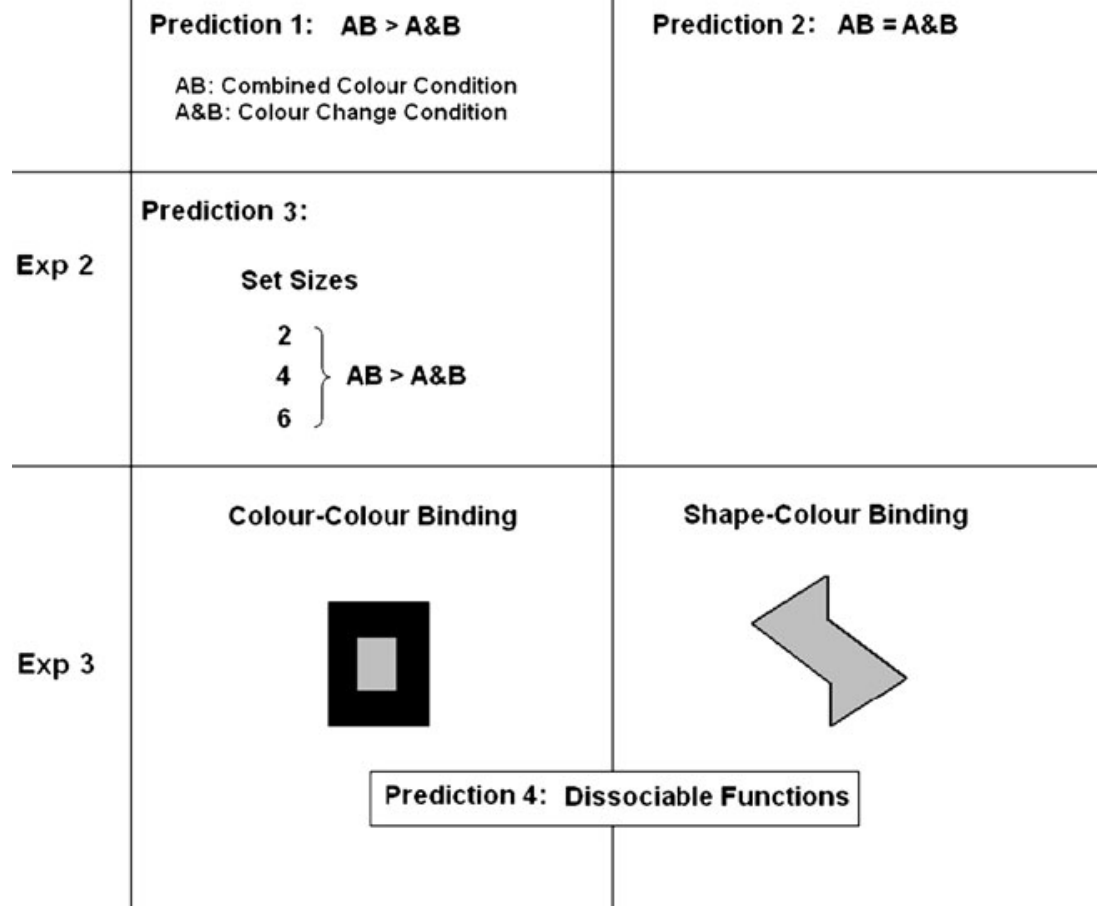

to test the hypothesis regarding the lack of color integration that had been suggested previously (Parra, Abrahams et al., 2009; Parra, Della Sala et al. 2009; Wheeler \& Treisman, 2002). If color parts of bicolored objects are retained in VSTM as separate entities, and not as features bound into unified representations, different patterns of performance would be expected when either part of bicolored objects changes (e.g., more hits or misses for a given part).

\section{Method}

Participants Twenty-six healthy young volunteers participated in Experiment 1 (age, $\mathrm{M}=20.27, \mathrm{SD}=1.31$; years of education, $\mathrm{M}=14.81, \mathrm{SD}=1.23$; sex, $\mathrm{M}=7, \mathrm{~F}=19)$. All gave their written consent prior to participation.

Stimuli Stimuli consisted of objects with an internal and anexternal area. Each areacomprised $50 \%$ of the surface of the object and was filled with one of eight colors (for RGB values, see http://www.era.lib.ed.ac.uk/bitstream/1842/2441/1/08278-MAP.doc). Four objects were presented in random positions on a 15 -in. PC screen using a $3 \times 3$ virtual grid. To construct the visual arrays for each trial, one object was selected from the set used by Parra, Abrahams et al. (2009); Parra, Della Sala et al. (2009). At the viewing distance of $65 \mathrm{~cm}$, objects subtended $0.75^{\circ}$, and the minimum distance between them was $0.5^{\circ}$.

Design and procedure Using a change detection task, participants were requested to judge two consecutivedisplays (i.e., study and test), which presented the stimuli described above. The design of the change detection task and its experimental conditions are shown in Fig.2. For each condition,on half of the trials, the objects in both displays were the same (same trials). On the other half, two of the objects in the test display showed colors different from those in the study display (different trials). Color changes were either in the internal area $(50 \%)$ or in the external area (50\%). Participants were requested to respond "different" or "same," depending on whether or not they detected changes between displays.

Two memory conditions were assessed (Fig. 2). In the color change condition, the external and the internal areas of each object were shown in different colors. On the different trials, one color either from the internal or from the external 


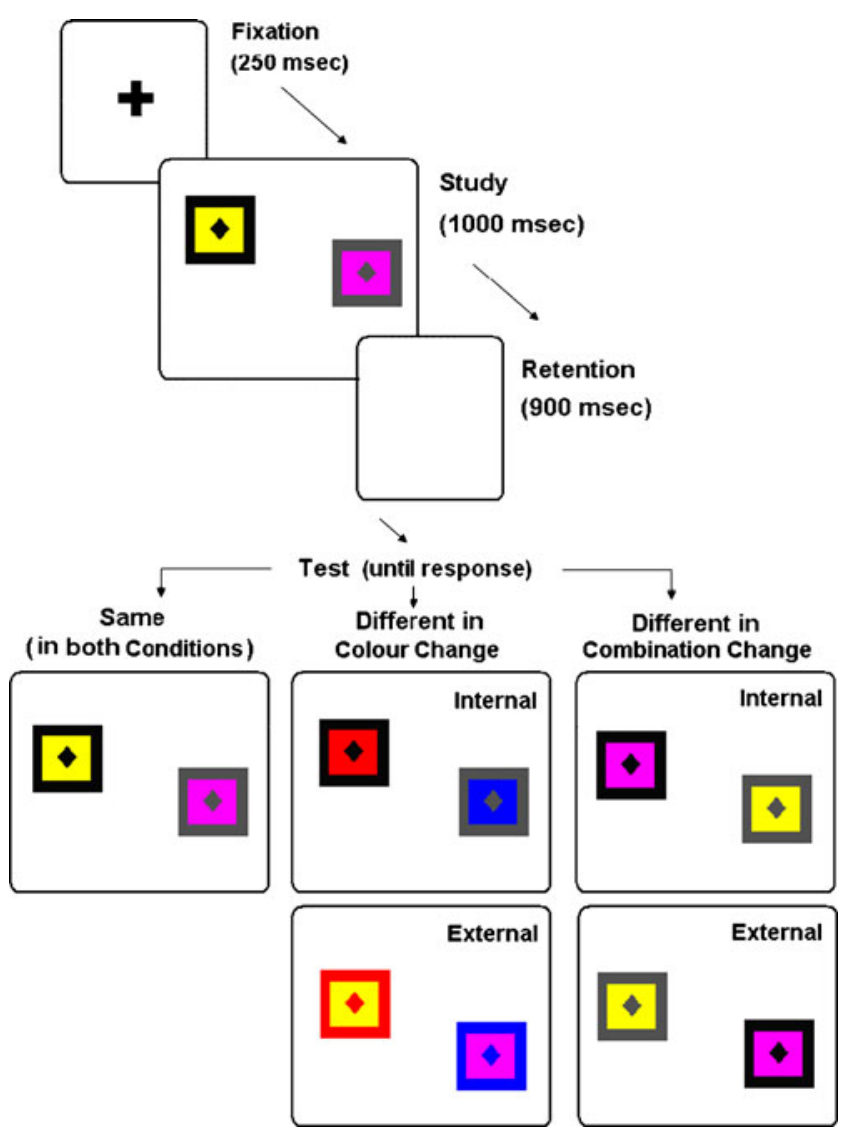

Fig. 2 Experimental procedure used to assessvisual short-term memory for color combinations (Combination Change) and for noncombined colors (color change). The set size shown is for illustration purposes.A presentation time of $1,000 \mathrm{~ms}$ was chosen since previous studies have suggested that shorter times may yield near floor performance (Parra, Abrahams et al., 2009; Parra, Della Sala et al., 2009).

area in two of the objects was replaced by a new color that had not appeared in the study display. The colors of the other two objects remained the same. Participants were told to focus on colors and not on their associations,since the change would consist of new colors. In the combined colors condition, both the internal and the external areas were of different colors. On the different trials, two objects swapped one color either from the external or from the internal area. The colors of the other two objects remained the same. Participants were told that colors and their associations were both relevant,since sometimes colors would be rearranged in different combinations during the test display. For the color change and combined colors conditions, colors could be repeated no more than twice within a display, but not within objects.

Participants performed 15 practice trials, followed by 48 trials, out of which 24 were same and 24 were different. The same and different trials were fully randomized, and conditions were blocked and counterbalanced across participants.
Analysis Two dependent variables were computed for Experiment 1:percentage of correctrecognition, defined as (hits/no. of different trials) $* 100$ (i.e., hits $=$ changes correctly recognized when either area changed), and response bias (beta). Response bias (beta) in a yes/no or same/different task is often quantified on the basis of a ratio of the probability of hits and the probability of false alarms (probability(hits) + probability(false alarms))/2; Stanislaw \& Todorov, 1999). Using the natural logarithm of this ratio, negative values of $\ln$ (beta) indicate bias toward no/same responses (rejecting the change; hence, more misses or correct rejection), whereas positive values of $\ln$ (beta) indicate bias toward yes/different responses (accepting the change; hence, more hits or false alarms). A value of 0 indicates no response bias (McNicol, 1972). Percentage of correct recognition would be informative of accuracy in remembering either color combinations or individual colors. Response bias would reveal any preferential processing across memory conditions and type of changes. For both variables, a two-way repeated-measures ANOVA was carried out. The repeated measures were condition (color change vs. combined colors) and change (external vs. internal).

Results

Percentage of correct recognition Mean performance data are shown in Fig.3a. A significant main effectwas found for condition, $F(1,25)=42.47, p<.001$, whereby combination change resulted in worse performance than did color change. Change also resulted in a significant main effect, $F(1,25)=5.78, p<.05$, whereby colors of the external area were remembered more accurately than colors of the internal area. The interaction of condition and change was found to be significant, $F(1,25)=5.79, \mathrm{p}<.05$. Pairedsamples $t$-tests showed that the interaction was driven by better memory performance for external color changes, relative to internal color changes, in the combination change condition, $t(25)=2.41, p<.05$, as compared with the color change condition.

Response bias (beta) Mean response bias is shown in Fig.3b. A significant main effect was found for condition, $F(1,25)=10.25, p<.01$, whereby combination change resulted in more preference for the sameresponse (i.e., more negative beta) than did color change. Change also resulted in a significant main effect, $F(1,25)=5.11, p<.05$, whereby bias for same responses was more pronounced for colors presented in the internal area than for colors presented in the external area. The interaction of condition and change was nonsignificant, $F(1,25)=0.18$, n.s., suggesting that the negative bias for internal colors was similar in both conditions. 
Fig. 3 a Mean percentage of correct recognition and $\mathbf{b}$ response bias (beta) in the two experimental conditions (color change and color combination) across the type of changes (internal and external).Error bars represent the standard errors of the means
(A)

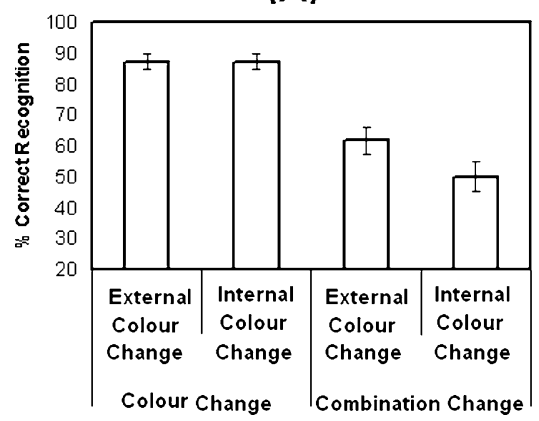

(B)

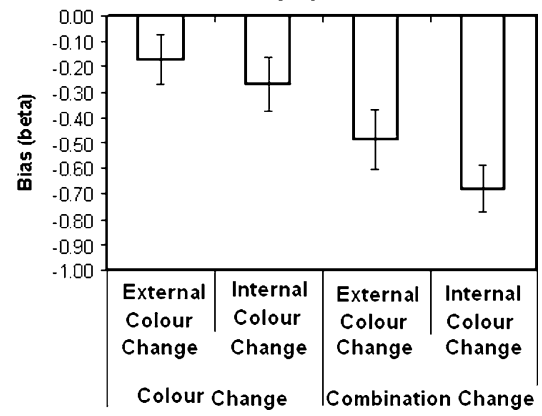

Comments

Remembering combinationsof colors was a more demanding task than remembering the same number of individual colors (see also Olson \& Jiang, 2002; Parra, Abrahams et al., 2009; Parra, Della Sala et al., 2009; Wheeler \& Treisman, 2002; Xu, 2002). Notably, colors presented in the external area were remembered better than colors presented in the internal area only in the combination change condition. These results provide direct evidence that colors are not integrated into single object representations in VSTM but are held as associations of individual features. It is worth noting that the differences across conditions could not be accounted for by saliency effects (see Table S1 in the Supplementary Material).

These results are challenging. The independent-stores hypothesis would support the prediction that colors that are processed within a single dimension but are presented as different parts of multicoloured objects will yield increased errors in changing trials in the combination change condition (i.e., as each part is processed separately). However, this hypothesis would not lend support to a prediction on a pattern of preference (i.e., bias) for external or for internal colors. The analysis of response bias sheds some light on this pattern of performance. It seems that participants are more likely to retain colors presented in the external than those presented in the internal area of bicolored objects. This makes changes in the internal area more likely to be missed (i.e., negative beta), providing evidence in favor of the lack of color integration in VSTM.

The better accuracy for external colors, together with a bias toward detecting changes in this area, supports the hypothesis that colors of bicolored objects are held in memory as two independent and associated, rather than integrated, pieces of information. If this is the case, retaining two colors together should require more memory capacity than retaining the same number of colors that have to be remembered individually. This hypothesis could be inferred from the outcomes of Experiment 1; however, it cannot be supported by these data. Differences in performance across conditions and type of changes suggest that capacity limitations may underpin the significant effects observed. Experiment 1 does not allow us to assess whether the procedures enable participants to group items into higher order chunks (e.g., remembering colors in the combination change as unified items, instead of as the association of individual colors), which is an important index of STM capacity as posited by Cowan (2001).

This hypothesis needs further investigation. First, memory performance in Experiment 1 was assessed at the level of VSTM capacity (i.e., four items; see Cowan, 2001; Vogel et al., 2001). Second, memory accuracy expressed as the percentage of correct recognition informs us about the demands of the tasks on the assessed functions but is not a direct measure of memory capacity (see Cowan, 2001). Third, in Experiment 1, the location of items was kept constant across study and test displays. Participants could use location as a memory cue (Treisman \& Zhang, 2006), which made this piece of information relevant to the task and likely to be bound to the remembered items.

\section{Experiment 2}

Experiment 2 investigated whether the lack of color integration previously suggested by other authors (Olson \& Jiang, 2002; Parra, Abrahams et al., 2009; Parra, Della Sala et al., 2009; Wheeler \& Treisman, 2002) and directly assessed for the first time in Experiment 1 could also be unveiled through the assessment of capacity limitations in VSTM. Our prediction was that if in order to correctly remember the bicolored objects, the link between colors should be retained in memory, in addition to the constituent colors, the effect of this additional cost should be observed regardless of the number of objects that have to be remembered. (see Fig. 1).

\section{Method}

Participants Twenty-eight new healthy young volunteers took part in Experiment 2 (age, $\mathrm{M}=21.68, \mathrm{SD}=1.49$; years of education, $\mathrm{M}=17.19, \mathrm{SD}=2.12$; sex, $\mathrm{M}=13, \mathrm{~F}=15$ ). All gave their written consent prior to participation. 
Design and procedure For Experiment 2, we used the same task as that devised by Parra, Abrahams, et al. (2009); Parra, Della Sala, et al. (2009), whichdiffered from the task used in Experiment 1 in the following ways: (1) The location of the items randomly changed from the study to the test display, and (2) the set sizes were two, four, and six. The other task parameters and conditions were the same as those in Experiment 1. As in Experiment 1, participants were told to focus on colors, and not on their associations, in the color change condition, or on colors and their associations, in the combined colors condition. Each condition (color change and combined colors) consisted of 32 trials, out of which 16 were same and 16 were different. Conditions were blocked and counterbalanced across participants. The same and different trials were fully randomized. This design did not allow assessing memory for parts of bicolored objects (i.e., external and internal), since this manipulation becomes irrelevant in the combined colors condition when objects change their spatial locations. For the color change condition, the design was the same as that described in Experiment 1. However, because the main finding of Experiment 1 concerned the combined colors condition and the main hypothesis for Experiment 2 concerned VSTM capacity as an additional underpinning of the lack of color integration in that condition, the effect of change (i.e., external area vs. internal area) was not considered in Experiment 2.

Analysis To assess the hypothesis set forth in this experiment, we chose the approach devised by Pashler (1988) and used by Luck and Vogel (1997), which considers the capacity of VSTM as the number of items effectively retained as a function of the total number of items toberemembered (set size), also controlling for the effects of guessing (i.e., false alarm rate). Following this approach, VSTM capacity was computed using the following expression: $K=[S *(H F)] /(1 F)$, where $K$ is capacity, $S$ is the set size, $H$ is the observed hit rate $(H=$ hits/[hits + misses] $)$, and $F$ is the observed false alarm rate $(F=$ false alarms/ [false alarms + correct rejections]). Capacity $(K)$ was analyzed using a two-way repeated-measures ANOVA with condition (color change vs. combined colors) and set size (2 vs. 4 vs. 6) as the within-subjects factors.

\section{Results}

Mean capacity data are shown in Fig.4. There was a significant effect of condition, $F(1,27)=21.42, p<.001$, whereby VSTM capacity was smaller for combination change than for color change. Set size also resulted in a significant main effect, $F(2,54)=13.53, p<.001$, whereby VSTM capacity increased as the memory load increased (all

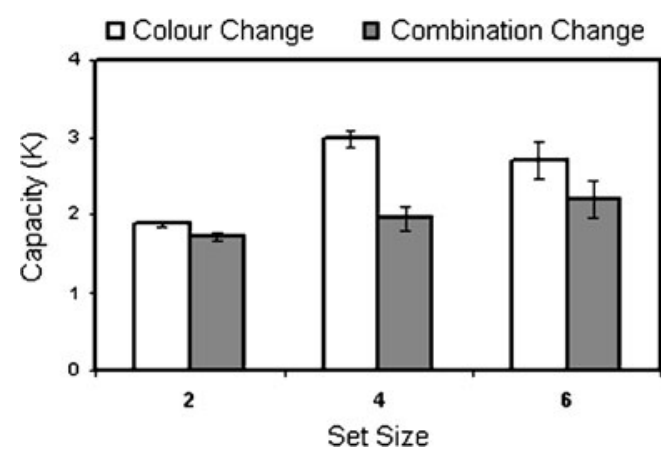

Fig. 4 Mean capacity in the two experimental conditions (color change and color combination)across the three set sizes.Error bars represent the standard errors of the means

$p$ s $<.001$ ), except for set size 6 , as compared with set size 4 . The condition $\times$ set size interaction was also significant, $F(2,54)=2.74, p<.05$. Further assessment of the interaction revealed that it was driven by a reduced memory capacity for combination change at set sizes 2 and 4, but not at set size 6 . The results showed that two seems to be the maximum number of bicolored objects that one can hold in VSTM when colors have to be remembered together.

\section{Comments}

More capacity was needed to retain color combinations in VSTM even when only two items were presented. At set size 4 , the cost of remembering how colors combined at study (i.e., combination change condition) exceeded that required to retain one bicolored object ( $K$ for combination change $=1.95$ and $K$ for color change $=2.99)$. This additional capacity should be that required to retain the information about how colors were combined. When the capacity of VSTM was exceeded (i.e., set size 6), the cost for the combination change condition was greater than that for the color changecondition, although not significantly. Hence, once the capacity of VSTM is overloaded, retaining the information on how colors combined becomes more challenging, and performance in the combination change condition rapidly deteriorates (see Parra, Abrahams et al., 2009; Parra, Della Sala et al., 2009; Wheeler \& Treisman, 2002; see also the Supplementary Material for accuracy data).It might be possible that under this condition, the participants chose to focus on a subset of items in order to alleviate the extra load imposed by the need to retain the binding between the colors of the items chosen.It is worth mentioning that the experimental manipulation introduced in Experiment 2 to make item location random could not account for the effects observed in Experiment 1 (see Table $\mathrm{S} 2$ in the Supplementary Material). These findings demonstrate that colors that have to be retained within bicolored objects in VSTM are not represented as integrated units but 
are remembered as two separate pieces of information that are associated within complex experiences. Differential memory accuracy for color parts of bicolored objects, a processing bias for external colors, and an additional capacity required to store the association between colors within bicolored objects seem to underpin the lack of color integration.

Taking the results of the present study and those of previous studies together, we suggest that binding information in VSTM across the shape and color dimension and within the color dimension are functions supported by different processes. Binding shape-color information leads to the formation of integrated units, the retention of which requires no more resources than those required to retain shapes only (Brockmole et al., 2008; Gajewski \& Brockmole, 2006; Luck \& Vogel, 1997; Vogel et al., 2001). Holding associations of colors within bicolored objects in VSTM requires considerably more capacity than does holding the same number of individual colors. This evidence suggests that the two processes may reflect the function of different memory mechanisms. If this were the case, we could predict dissociations for shape-color and color-color binding in VSTM (see Fig. 1). In Experiment 3, we investigated this hypothesis in E.S., a brain-damaged patient who presents with profound impairments to process shape-color bindings in VSTM. The case of this patient is fully reported in Parra, Della Sala et al. (2009).

\section{Experiment 3}

Experiment 3 investigated whether the type of representational format (i.e., conjunctions vs. associations) used to retain multifeature objects in VSTM may differ according to the material to be bound. On the basis of the data from Experiments 1 and 2, we would predict that a differential impairment may be observed for memory representations based either on integrative mechanisms (i.e., shape-color) or on associative mechanisms (i.e., color-color). If this hypothesis were valid, it would be possible to observe dissociations within VSTM for within- and across-dimension binding in individuals with brain damage.Since E.S. had already showed a severe impairment in cross-dimension binding, it was useful to investigate whether within-dimension binding remained spared in this patient.

Method

Participants E.S. and 10 healthy controls matched with E. S. for age (E.S., 69; controls, $M=67.4, S D=6.41 ; t=0.24$, n.s.) and years spent in formal education (E.S., 10; controls, $M=14.1, S D=2.68 ; t=1.26$, n.s.) participated in Experiment 3. E.S. was a right-handed woman who had a meningioma in the left wing of the sphenoid, which,before surgery, impinged upon her left temporal lobe. She showed normal performance in a large neuropsychological battery assessing memory, attention, executive, and language functions. However, E.S.'s VSTM for shape-color bindings was dramatically impaired. A full clinical and neuropsychological profile of E.S. is detailed in Parra, Della Sala, et al. (2009). A summary of her neuropsychological assessment is also presented in the Supplementary Material (Table S3).

Design and procedureE.S. and controls performed the same task as that described in Experiment 2, including setsizes 2 and 4. Performance of E.S. and controls on the shape-color binding task was assessed using the same task as that described by Parra, Della Sala, et al.'s Experiment 1 (2009). In this task, shape-color bindings are presented in both displays. On the different trials, two shapes swapped their colors in the test display. Participants were requested to report whether or not they detected a change between displays (see Parra, Della Sala, et al., 2009, for a full description of this task). Set size 6 was not included in Experiment 3,because E.S.'s performance on the shape-color binding task with this set size was at floor. All participants gave their written consent prior to participation.

Analysis Percentage of correct recognition and VSTM capacity $(K)$ were computed. Set sizes 2 and 4 only from the combination change condition were used.

To compare the performance of E.S. and controls, we used the statistics devised by Crawford and Garthwaite (2002) to investigate selective impairments in single-case studies. We calculated the point estimate $(\mathrm{PE})^{1}$ of abnormality, which indicates the percentage of the control population that would be expected to obtain a score lower than that of E.S. Then we assessed whether the pattern of performance analyzed across forms of binding fulfilled the criteria for classical dissociation (Crawford \& Garthwaite, 2005b; Crawford, Garthwaite, \& Gray, 2003). For all statistics, we report the PE, one-tailed $t$-values (a poorer performance by E.S., relative to controls, was predicted), and the probability that E.S.'s scores fell outside the normal range.

\footnotetext{
${ }^{1}$ Crawford and Garthwaite (2002) developed a method for obtaining a $\mathrm{PE}$ of the abnormality of a difference between a patient's score and the average score of a modest sample that treats the normative sample statistics as statistics, rather than as parameters. This is achieved by obtaining a PE of the abnormality of a pair of test scores.
} 
Results

Percentage of correct recognition Fig.5a shows the mean percentages of correct recognition for E.S. and controls across types of binding and set sizes (see Table S4 in the Supplementary Material for the descriptive statistics). When E.S.'s and the controls' scores in the shape-color binding condition were compared, E.S. showed a clear impairment with both set sizes $[2, \mathrm{PE}=1.38, t(9)=2.62, p=.014 ; 4$, $\mathrm{PE}=0.05, t(9)=4.74, p<.001]$. No significant differences were observed when E.S.'s and controls' scores were compared across set sizes in the color-color binding task [set size $2, \mathrm{PE}=6.1, t(9)=1.7, p=.06$; set size $4, \mathrm{PE}=$ $31.9, t(9)=0.49$, n.s.].

VSTM capacity (K) Fig.5b shows mean capacity data for E. S. and controls across types of binding and set sizes. When E.S.'s and controls' capacity scores in the shape-color binding condition were compared, E.S. showed a dramatic reduction in her VSTM capacity at both set sizes [2, PE $=$ $0.00, t(9)=13.63, p<.001 ; 4, \mathrm{PE}=0.00, t(9)=6.82, p<$ $.001]$. No one from the normal population would be expected to show smaller VSTM capacity than E.S. No significant differences in capacity were observed when E. S.'s and controls' scores were compared across set sizes in the color-color binding task $[2, \mathrm{PE}=18.3, t(9)=0.96$, n.s.; $4, \mathrm{PE}=32.0, t(9)=0.49$, n.s. $]$.

Assessing dissociations in VSTM The outcome from the analyses above suggests that VSTM for shape-color bindings could be impaired in the presence of preserved color-color binding functions. This supports the hypotheses originating Experiment 3. However, in order to assess whether this pattern of impairment fulfills the classical criteria for dissociation, we entered these data into the formula devised by Crawford and Garthwaite (2005a). The outcome shows a $\mathrm{PE}=0.05, t(9)=4.72, p<.001$, indicating that E.S.'s pattern of performance fulfilled the criteria for a classical simple dissociation.
Comments

In Experiments 1 and 2, we have shown that the processes responsible for binding features within and across dimensions in VSTM seem to be supported by different cognitive mechanisms. In contrast to shape-color binding (Brockmole et al., 2008; Gajewski \& Brockmole, 2006; Luck \& Vogel, 1997; Vogel et al., 2001), color-color binding does not lead to the formation of a unified representation (Experiment 1) and requires additional capacity to retain the relation between features within objects (Experiment 2). The segregated nature of these memory operations was further supported by the outcomes of Experiment 3, which reported the case of a patient showing a dramatic impairment in binding shape with colors in VSTM, whereas her performance in binding colors within bicolored objects was preserved. It is worth noting that color-color binding is the more demanding of the two tasks (see the Introduction) and that, in the paradigm used in Experiment 3, healthy participants do not find color-color binding overall easier to perform than shape-color binding, as evidenced by their performance (see Fig.5).

\section{General discussion}

The results from the series of experiments presented here confirm the hypotheses that colors are not integrated in VSTM into unified objects (Olson \& Jiang, 2002; Parra, Abrahams et al., 2009; Parra, Della Sala et al., 2009; Wheeler \& Treisman, 2002; Xu, 2002) but are, rather, retained as associations in VSTM. Three different sets of evidence support this proposal. They will be discussed in turn.

\section{Memory bias}

The observation of memory superiority for color parts of bicolored objects (i.e., external colours remembered better
Fig. 5 a Mean percentage of correct recognition and $\mathbf{b}$ capacity $(K)$ for E.S. and controls across types of binding and set sizes.Error bars represent the standard errors of the means

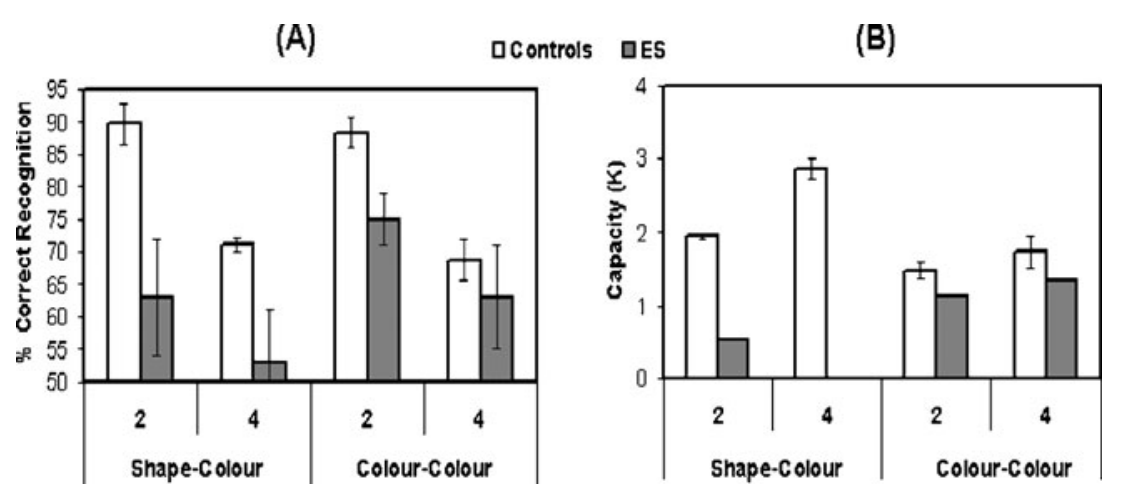


than internal colors) offers evidence in support of a lack of color integration in VSTM. We predicted that, if colors are represented in VSTM not as integrated units, but as individual entities, each color of the bicolored objects could be differentially remembered. We did not assume, however, that the differential memory for color parts would reflect a processing bias for external colors. Should the independent-stores hypothesis hold true for bicolored objects, one would expect to observe differential performance for external or internal colors, but not necessarily a consistent pattern for either part, as was observed in Experiment 1.

There is no account available in the VSTM literature to explain this processing bias for external colors in the combination change condition. Considerable evidence, however, could be gleaned from the memory and perception literature concerning figure/background representation. If we were to assume that the external colors of our stimuli could be interpreted as background colors, the effect appears to be consistent with evidence from the literature on memory (Isarida \& Isarin, 2007; O'Herron \& von der Heydt, 2009; Peterson \& Grant, 2003) and perception (Hecht \& Vecera, 2007; Vecera, 2004; Vecera \& Palmer, 2006; but see Lester, Hecht, \& Vecera, 2009). According to this literature, background information plays a crucial role in forming and accessing complex representation in memory and perception. Should these organizational principles also apply to VSTM, one might suggest that the background-processing superiority reported in previous studies and the memory superiority for external colors of bicolored objects observed in the present study may reflect the outcomes of related functions.

The memory superiority for external colors allowed us to empirically demonstrate that colors are not integrated into inseparable units. However, further research is needed to characterize this processing bias. Future studies should investigate this processing bias, using recall instead of recognition paradigms, whereby participants would be asked to report the changing area/color (i.e., external or internal) during combination change and color change, instead of recognizing changes occurring in whole arrays.

\section{Capacity limitations}

The tasks used in the present study enabled the assessment of the extra capacity required to retain color bindings in VSTM,since the color change condition provideda clear cut baseline performance. Since combination change and color change conditions presented exactly the same amount of information, performance drop in the combination change condition could be interpreted as the need for retaining the additional information on how colors combined (i.e., the binding). This was found to be case when the number of items did not overload the memory capacity. In fact, the binding between colors within bicolored objects seems to be a function supported by processes other than those involved in color processing. For example, in Experiment 2, the VSTM capacity in the combination change and color change conditions was greater than two items and was at the same level with set size 6. The amount of color information to be processed overloaded the capacity of this single dimension (Olson \& Jiang, 2002; Parra, Abrahams et al., 2009; Parra, Della Sala et al., 2009; Wheeler \& Treisman, 2002; Xu, 2002). However, the finding that performance was above chance in both conditions (see Table S5, Supplementary Material) suggests that the binding operations required to perform the combination change condition, which we have demonstrated to require extra resources, were not supported by the same mechanisms as those involved in color processing. The data presented here suggest that resources within the color dimension were depleted more in the combination change condition than in the color change condition. Hence, to achieve above-chance performance in the combination change condition, resources other than those provided by the color dimension should have been used.

Recent fMRI studies suggest that individual and bound features are processed in different brain regions. Prefrontal regions seem to be important for binding functions (Prabhakaran, Narayanan, Zhao, \& Gabrieli, 2000), whereas posterior parietal regions seem to be involved in feature processing (Xu, 2007). Furthermore, there is currently a debate in the literature regarding the conditions in which attention is required for shape-color bindings, with recent studies demonstrating an involvement of attention (Brown \& Brockmole, 2010; Elsley \& Parmentier, 2009). However, recent studies suggest that binding shapes and colors within single items in working memory does not require more attentional resources than those required to process individual features (see Allen, Baddeley, \& Hitch, 2006; Allen, Hitch, \& Baddeley, 2009; Johnson, Hollingworth, \& Luck, 2008). This evidence, together with the results presented here, supports the proposal that whereas shape-color bindings could be processed automatically in VSTM, the link between colors within bicolored objects has to be actively maintained in order to accurately remember these complex stimuli.

\section{Dissociation within VSTM binding and its implications}

If the link between colors within bicolored objects has to be actively retained in VSTM in order to accurately remember the objects, these binding processes would be different from those we have been presented with in literature supporting the object-based hypothesis of VSTM (Allen et al., 2006; Brockmole et al., 2008; Gajewski \& Brockmole, 2006; 
Luck \& Vogel, 1997; Vogel et al., 2001). This literature suggests that in the unified object representation, bound features lose their individual properties and adopt those defining the new object. Hence, remembering only one feature would not grant access to the other features that belong to a specific object representation. If we were to interpret this as a conjunction of features in VSTM, the present data with color-color binding would be interpreted as a relation of features in VSTM.

There seem to be similarities between the functional constructs of the object-unit hypothesis and the independentstores hypothesis proposed by Gajewski and Brockmole (2006) and contrasted in the present study and the conjunctive and relational hypotheses discussed by Moses and Ryan (2006). The first two hypotheses have been postulated in order to investigate working and short-term memory binding functions, whereas the second two hypotheses mainly concern long-term memory binding functions. The conjunctive hypothesis proposes that features are bound into integrated units (e.g., features within faces, colors within shapes) in a way that leads to changes in objects' identity (similar to the object-unit hypothesis). The relational hypothesis suggests that features are associated within complex memory networks; however, each retains its own identity (similar to the independent-stores hypothesis). The outcomes from previous studies investigating VSTM performance with shape-color bindings suggest that the predictions of the conjunctive and the object-based hypotheses could both account for this VSTM function (Allen et al., 2006; Brockmole et al., 2008; Gajewski \& Brockmole, 2006; Vogel et al., 2001). The results presented here suggest that VSTM performance with color-color bindings could be accounted for by the independent-stores hypothesis, whose predictions are similar to those of the relational hypothesis. That is, colors are not bound into single objects, but their relation within objects is held in memory when the task requires such an association.

Taken together, the data collected from E.S. in a previous study (Parra, Della Sala et al., 2009) and in the present study suggest that shape-color bindings and color-color bindings are stimuli processed in VSTM via different mechanisms. Parra, Della Sala, et al. proposed that the binding between shapes and colors represents an additional piece of information that seems to be processed by mechanisms distinct from those responsible for feature processing and feature memory. Current data from healthy individuals with color-color bindings also support this view, and in the case for E.S., they suggest that this form of memory representation is processed by mechanisms other than those responsible for processing shape-color bindings. Color-color bindings are processed in VSTM by mechanisms that may be more accurately accounted for by the independent-stores hypothesis (or relational hypothesis), whereas shape-color bindings seem to be represented via mechanisms more linked to the predictions of the object-based hypothesis (or conjunctive hypothesis). The scarce literature available on VSTM and working memory binding suggests that holding conjunctions of features (i.e., shape-colors) and holding relations between features as associations in memory are processes supported by different mechanisms and brain regions (Baddeley, Allen, \& Vargha-Khadem, 2010; Parra et al., 2010; Parra et al., 2011; Piekema, Kessels, Mars, Petersson, \& Fernandez, 2006). Baddeley et al. assessed Jon, a patient with developmental amnesia whose pathology appears to be limited to the hippocampus. Jon showed preserved working memory functions for shape-color binding, while E.S.'s presented with a severe impairment. E.S.'s lesion impinged upon the anterior pole of the left temporal lobe. It might be possible that medial temporal lobe structures other than the hippocampus could be involved in this form of binding. This, together with the data presented here, supports the idea that some of the properties of binding processes in VSTM are dissociable.

\section{Conclusions}

The results presented here provide novel evidence that can help to explain why color-color bindings (i.e., withindimension binding) are retained in VSTM less efficiently than shape-color bindings (i.e., across-dimensions binding). Color combinations are subserved by associative memory mechanisms, while shape-color combinations are subserved by conjunctive mechanisms. These mechanisms seem to pose different memory demands and are differentially vulnerable to brain damage.

Acknowledgments We thank Louise Brown and James R. Brockmole for their valuable comments on earlier drafts of our manuscript. M.A.P. and R.C. are supported by a grant from the Neuroscience Program of the "San Paolo Foundation."

\section{References}

Allen, R. J., Baddeley, A. D., \& Hitch, G. J. (2006). Is the binding of visual features in working memory resource-demanding? Journal of Experimental Psychology: General, 135, 298-313.

Allen, R. J., Hitch, G., \& Baddeley, A. (2009). Cross-modal binding and working memory. Visual Cognition, 17, 83-102.

Baddeley, A., Allen, R., \& Vargha-Khadem, F. (2010). Is the hippocampus necessary for visual and verbal binding in working memory? Neuropsychologia, 48, 1089-1095.

Brockmole, J. R., Parra, M. A., Della Sala, S., \& Logie, R. (2008). Do binding deficits account for age-related decline in visual working memory? Psychonomic Bulletin \& Review, 15, 543-547.

Brown, L. A., \& Brockmole, J. R. (2010). The role of attention in binding visual features in working memory: Evidence from cognitive ageing. Quarterly Journal of Experimental Psychology, 63, 2067-2079. 
Cowan, N. (2001). The magical number 4 in short-term memory: a reconsideration of mental storage capacity. Behavioral and Brain Sciences, 24, 87-114.

Crawford, J. R., \& Garthwaite, P. H. (2002). Investigation of the single case in neuropsychology: confidence limits on the abnormality of test scores and test score differences. Neuropsychologia, 40, 1196-1208.

Crawford, J. R., \& Garthwaite, P. H. (2005a). Evaluation of criteria for classical dissociations in single-case studies by Monte Carlo simulation. Neuropsychology, 19, 664-678.

Crawford, J. R., \& Garthwaite, P. H. (2005b). Testing for suspected impairments and dissociations in single-case studies in neuropsychology: Evaluation of alternatives using Monte Carlo simulations and revised tests for dissociations. Neuropsychology, 19, 318-331.

Crawford, J. R., Garthwaite, P. H., \& Gray, C. D. (2003). Wanted: fully operational definitions of dissociations in single-case studies. Cortex, 39, 357-370.

Elsley, J. V., \& Parmentier, F. B. (2009). Is verbal-spatial binding in working memory impaired by a concurrent memory load? Quarterly Journal of Experimental Psychology, 62, 1696-1705.

Gajewski, D. A., \& Brockmole, J. R. (2006). Feature bindings endure without attention: Evidence from an explicit recall task. Psychonomic Bulletin \& Review, 13, 581-587.

Hecht, L. N., \& Vecera, S. P. (2007). Attentional selection of complex objects: joint effects of surface uniformity and part structure. Psychonomic Bulletin \& Review, 14, 1205-1211.

Isarida, T., \& Isarin, T. K. (2007). Environmental context effects of background color in free recall. Memory \& Cognition, 35, 1620 1629.

Johnson, J. S., Hollingworth, A., \& Luck, S. J. (2008). The role of attention in the maintenance of feature bindings in visual short-term memory. Journal of Experimental Psychology: Human Perception and Performance, 34, 41-55.

Lester, B. D., Hecht, L. N., \& Vecera, S. P. (2009). Visual prior entry for foreground figures. Psychonomic Bulletin \& Review, 16, 654-659.

Luck, S. J., \& Vogel, E. K. (1997). The capacity of visual working memory for features and conjunctions. Nature, 390, 279-281.

McNicol, D. (1972). A primer of signal detection theory. London: Allen \& Unwin.

Moses, S. N., \& Ryan, J. D. (2006). A comparison and evaluation of the predictions of relational and conjunctive accounts of hippocampal function. Hippocampus, 16, 43-65.

O'Herron, P., \& von der Heydt, R. (2009). Short-term memory for figure-ground organization in the visual cortex. Neuron, 61, 801-809.

Olson, I. R., \& Jiang, Y. (2002). Is visual short-term memory object based? Rejection of the "strong-object" hypothesis. Perception \& Psychophysics, 64, 1055-1067.
Parra, M. A., Abrahams, S., Logie, R., \& Della Sala, S. (2009). Age and binding within-dimension features in visual short term memory. Neuroscience Letters, 449, 1-5.

Parra, M., Abrahams, S., Logie, R., Mendez, L. G., Lopera, F., \& Della Sala, S. (2010). Visual short-term memory binding deficits in familial Alzheimer's disease. Brain, 133, 2702-2713.

Parra M. A., Della Sala, S., Abrahams, S., Logie, R.H., Mendez, L.G., \& Lopera, F. (2011). Specific deficit of colour-colour short-term memory binding in sporadic and familial Alzheimer's disease. Neuropsychologia. doi:10.1016/j.neuropsychologia.2011.03.022

Parra, M. A., Della Sala, S., Logie, R., \& Abrahams, S. (2009). Selective impairment in visual short-term memory binding. Cognitive Neuropsychology, 26, 583-605.

Pashler, H. (1988). Familiarity and visual change detection. Perception \& Psychophysics, 44, 369-378.

Peterson, M. A., \& Grant, M. S. (2003). Memory and learning in figure-ground perception. In B. Ross \& D. Irwin (Eds.), Cognitive vision: Psychology of learning and motivation (42nd ed., pp. 1-34). San Diego, CA: Psychology Press.

Piekema, C., Kessels, R. P., Mars, R. B., Petersson, K. M., \& Fernandez, G. (2006). The right hippocampus participates in short-term memory maintenance of object-location associations. NeuroImage, 33, 374-382.

Prabhakaran, V., Narayanan, K., Zhao, Z., \& Gabrieli, J. D. (2000). Integration of diverse information in working memory within the frontal lobe. Nature Neuroscience, 3, 85-90.

Stanislaw, H., \& Todorov, N. (1999). Calculation of signal detection theory measures. Behavior Research Methods, Instruments, \& Computers, 31, 137-149.

Treisman, A., \& Zhang, W. (2006). Location and binding in visual working memory. Memory \&Cognition, 34, 1704-1719.

Vecera, S. P. (2004). The reference frame of figure-ground assignment. Psychonomic Bulletin \& Review, 11, 909-915.

Vecera, S. P., \& Palmer, S. E. (2006). Grounding the figure: Surface attachment influences figure-ground organization. Psychonomic Bulletin \& Review, 13, 563-569.

Vogel, E. K., Woodman, G. F., \& Luck, S. J. (2001). Storage of features, conjunctions and objects in visual working memory. Journal of Experimental Psychology: Human Perception and Performance, 27, 92-114.

Wheeler, M. E., \& Treisman, A. M. (2002). Binding in short-term visual memory. Journal of Experimental Psychology: General, 131, 48-64.

$\mathrm{Xu}, \mathrm{Y}$. (2002). Limitations of object-based feature encoding in visual short-term memory. Journal of Experimental Psychology: Human Perception and Performance, 28, 458-468.

$\mathrm{Xu}$, Y. (2007). The role of the superior intraparietal sulcus in supporting visual short-term memory for multifeature objects. Journal of Neuroscience, 27, 11676-11686. 